\title{
e-Accesibilidad: del enfoque centrado en el contenido al enfoque comunicacional
}

\author{
E-Accessibility: from content-centric approach to communicative approach
}

\author{
María Inés LAITANO \\ Laboratoire Paragraphe, Université Paris 8, 2 rue de la Liberté - 93526 Saint-Denis, ines.laitano@gmail.com
}

\begin{abstract}
Resumen
Se plantea que los estándares de accesibilidad tradicionales se focalizan en el contenido; y se propone, en respuesta, un enfoque comunicacional de la e-Accesibilidad donde la interfaz es medio de comunicación y de acción. El enfoque comunicacional encuentra sus fundamentos teóricos en modelos significativos del campo de la Interacción Persona-Ordenador, en particular aquellos que adoptaron la Semiótica y la Teoría de la Actividad como bases para estudiar el diseño de interfaz. Su originalidad radica en la integración de la dimensión discursiva al modelo de la accesibilidad. Tomando un portal que ofrece cursos abiertos en línea y masivos (en adelante MOOC) como caso de estudio, se comparan las soluciones de diseño accesible propuestas por el enfoque centrado en el contenido y por el enfoque comunicacional. Se muestra que el segundo puede complementar al primero en pos de una accesibilidad comunicacional más íntegra.
\end{abstract}

Palabras clave: e-Accesibilidad. Inclusión. Diseño de interacción. Interfaces de ordenador. MOOC. Semiótica. Teoría de la actividad. Dimensión discursiva.

\section{Introducción}

La e-Accesibilidad, es decir el derecho de acceso a la información digital más allá de las singularidades físicas, psíquicas, culturales, geográficas o económicas de las personas, juega un rol fundamental en la inclusión social. La oportunidad de acceder a una formación virtual, por ejemplo, puede mejorar potencialmente las condiciones de vida de una persona que encuentra barreras para desplazarse físicamente hasta el lugar de estudio. Existe sin embargo vasta evidencia de que la información digital, y la educación virtual en particular (García Gómez, 2010; Laitano, 2015a), no cuentan aún con niveles mínimos de accesibilidad.

El modo tradicional de evaluar la e-Accesibilidad es a través de pautas o estándares. Las pautas reconocidas actualmente como norma de facto en todo el mundo para evaluar la accesibilidad web son las Web Content Accessibility Guidelines (WCAG) 2.0 desarrolladas por la Web Accessibility Initiative (WAI), una iniciativa del World Wide Web Consortium (W3C). Cada pauta de las

\begin{abstract}
It is argued that traditional accessibility standards have a content-centric perspective and proposes, in response, a communicative approach of e-Accessibility where the interface is a mean of communication and action. The communicative approach finds its theoretical foundations in significant models from the Human Computer Interaction field; particularly in those that adopted Semiotics and Activity Theory as a basis for studying interface design. Its originality lies in integrating the discursive dimension to the accessibility model. A portal that offers massive open online courses (MOOC) is taken as a case study. Considering the accessible design solutions proposed for this particular case study, a comparison between the content-centric perspective and the communicative approach is made in order to show that the latter can complement the former in pursuit for a more complete communicative accessibility.
\end{abstract}

Keywords: Accessibility. Inclusion. Interaction design. Computer interfaces. MOOC. Semiotics. Activity theory. Discursive dimension.

WCAG 2.0 está compuesta por una serie de criterios de conformidad que pueden verificarse sin ambigüedades sobre una página web. Así, el nivel de accesibilidad de una página web depende, según la WAI, de la cantidad de criterios de conformidad que la misma respeta.

El presente artículo sostiene que el enfoque de la WAI es un enfoque centrado en el contenido que como tal omite ciertas dimensiones fundamentales de la comunicación mediada. Propone adoptar un enfoque más amplio que no se contente con garantizar la legibilidad a los contenidos sino que garantice una comunicación mediada accesible. Las secciones que siguen buscan justificar e ilustrar esta tesis, proponiendo una perspectiva original que recibe el nombre de enfoque comunicacional puesto que reflexiona sobre la interfaz como medio de comunicación y de acción. 


\section{El enfoque centrado en el contenido y sus limitaciones}

El artículo Interdependent components of web accessibility (Chisholm y Henry, 2005) describe el modelo que subyace al enfoque de la WAI. Para ellos la accesibilidad web depende de la interdependencia entre ciertos componentes técnicos y humanos que funcionan juntos (Figura 1). El contenido es el componente técnico central del modelo y se define como (1) (ibidem, p. 32):

[...] la información que constituye los sitios web y las aplicaciones web: el código y el marcado que definen la estructura, la presentación y la interacción, así como el texto, las imágenes y los sonidos que transmiten información al usuario final.

Entre los demás componentes técnicos se encuentran, por un lado, los que permiten crear contenido web como las herramientas de autor y de evaluación, y, por otro, los que permiten ver e interactuar con el contenido, a los que la WAI denomina agentes de usuario. Las herramientas de autor incluyen entornos de desarrollo, content management systems, aplicaciones de blog, etc. Las herramientas de evaluación son las que se utilizan para chequear la sintaxis y la accesibilidad de los contenidos. Los agentes de usuario incluyen los navegadores web, los reproductores multimedia y las tecnologías asistivas (2).

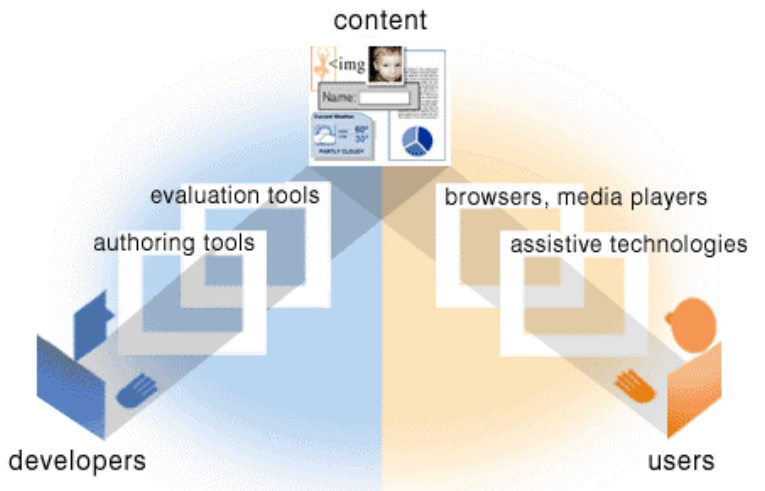

Figura 1. Modelo del enfoque centrado en el contenido (Duffy, 2016, w3.org/WAl/intro/components.php)

En cuanto a los componentes humanos, la WAI reconoce: los productores de contenido (desarrolladores en la Figura 1) que son quienes diseñan, programan y editan el contenido web; los desarrolladores de herramientas (ausentes en la Figura 1) que son quienes diseñan, programan y testean las herramientas de autor, de evaluación y los agentes de usuario; y por último los usuarios finales que son quienes usan los agentes de usuario para consultar e interactuar con el conte- nido web. Los usuarios finales tienen distintas habilidades, distintas vivencias y configuran los agentes de usuario de maneras diversas.

En este contexto, las WCAG 2.0 aparecen como recomendaciones técnicas destinadas a los productores de contenido que explican cómo crear contenido web accesible para los usuarios finales. Al centrarse en el contenido como componente técnico, es decir como texto, imagen, baliza de marcado, etc., las WCAG 2.0 pueden hacer recomendaciones de tipo universal. Por ejemplo, todo contenido no textual debe poseer una alternativa textual que persiga el mismo propósito (W3C, 2009, sec. 1.1.1). Tal como se mostrará más adelante y como lo plantean Kelly y otros (2009, pp. 266-267) para el caso de la publicidad o del arte, esta pauta no garantiza que el contenido sea comunicacionalmente accesible.

El enfoque de la WAI ha sido cuestionado desde diferentes perspectivas. Di Blas, Paolini y Speroni (2004) por ejemplo sostienen que las directrices de la WAI sólo garantizan la legibilidad técnica de una página web, esto es, que la página web pueda ser leída correctamente por las tecnologías de apoyo que usan las personas con discapacidad. La accesibilidad técnica no garantiza para los autores una experiencia de usuario efectiva, por lo que proponen considerar la accesibilidad como una rama de la usabilidad: un sitio web será usable si lo es también para las personas con discapacidad. En el mismo sentido, Pastor Sánchez (2010) cuestiona la imprecisión de las pautas en lo que refiere a la comprensibilidad y organización de los contenidos (arquitectura de la información). Kelly y otros (2009) introducen la noción de accesibilidad 2.0 para nombrar un nuevo enfoque que aborda las necesidades del usuario en lugar del cumplimiento de directrices. Para los autores, la accesibilidad no puede reducirse a la conformidad de un recurso digital con ciertas pautas técnicas (Sloan y Kelly, 2011). Es necesario adoptar un enfoque relacional: la accesibilidad sería una propiedad de la relación entre el usuario y el recurso digital en un contexto de uso, no una propiedad del recurso en sí (Cooper, Sloan, Kelly y Lewthwaite, 2012). Así, evaluar la e-Accesibilidad significaría evaluar principalmente aspectos de la experiencia de usuario.

Otros autores se han abocado a demostrar empíricamente que las pautas de la WAI no cubren la totalidad de los problemas encontrados por las personas con discapacidad cuando navegan por la Web. Rømen y Svanæs (2011) por ejemplo realizaron test controlados de usabilidad y encontraron que menos de la mitad de los problemas reportados por las personas con discapacidad se podrían haber identificado utilizando las WCAG. En consecuencia, propusieron que el concepto 
de accesibilidad de la WAI debería ampliarse al de usabilidad para todos. De la misma manera, Power, Freire, Petrie y Swallow (2012) demostraron, mediante tests de usuarios basados en tareas, que las WCAG 2.0 cubrían sólo la mitad de los problemas encontrados por las personas con ceguera. Estos autores también plantearon que la WAI debería inspirarse en el campo de la usabilidad y formular principios de diseño más amplios fundamentados en el uso de la Web que hacen las personas con discapacidad.

En la misma línea, sondeos realizados a usuarios de lector de pantalla (3) ponen en evidencia que las soluciones sugeridas por la WAI no son siempre las que los usuarios prefieren. Al ser consultados sobre las imágenes de la Web que buscan generar alguna emoción o sensación, el 59\% de los 1121 encuestados indicó que prefería que la imagen sea descripta por su lector de pantalla contra el $31 \%$ que respondió que la imagen debía ser ignorada por su lector de pantalla (WebAIM, 2009). Más aun, la gran mayoría de los que respondieron que la imagen debía ser ignorada eran usuarios sin discapacidad, es decir, usuarios que utilizan el lector de pantalla para evaluar la accesibilidad web. Este resultado se opone claramente al criterio de conformidad 1.1.1 de las WCAG 2.0, según el cual las imágenes decorativas no deben describirse, y deja ver ciertas carencias de comunicación que aún presenta la Web para las personas con discapacidad visual.

\section{El enfoque comunicacional propuesto}

El enfoque comunicacional que aquí se propone pretende enriquecer la perspectiva centrada en el contenido de la WAI. El enfoque encuentra sus fundamentos teóricos en modelos que fueron propuestos con anterioridad en el campo de la Interacción Persona-Ordenador. Particularmente, aquellos que adoptaron la Semiótica (Bootz, 2007; de Souza, 2005; Pignier y Drouillat, 2004; Scolari, 2009; Laitano, 2015b) y la Teoría de la Actividad (Kaptelinin, 2013; Rabardel, 1995) como bases para estudiar el diseño de la interfaz.

Desde una perspectiva semiótica, la interacción persona-ordenador puede ser vista como una comunicación entre un proyectista y un público objetivo mediada por la interfaz. El proyectista no designa únicamente a los productores de contenido como en el enfoque de la WAI sino a todas las personas que tienen un proyecto con respecto al público objetivo. Por ejemplo, en un sitio web que brinda cursos de tipo MOOC el proyectista está compuesto por las universidades que auspician los cursos, por los profesores que dictan los cursos y por los gestores de la informa- ción de los cursos. El público objetivo es el conjunto de sujetos que identifica y define el proyectista como potenciales usuarios de la interfaz. En el ejemplo, el público objetivo está compuesto por el grupo de personas que desean tomar cursos MOOC.

El proyectista tiene una estrategia de comunicación con respecto al público objetivo, es decir, una manera particular de organizar el diseño de la interfaz en vistas de alcanzar el proyecto que se propuso. En el ejemplo citado, el proyectista puede desear que el público tenga claros los objetivos del curso. Para ello ofrecerá, desde la página de inicio del curso, un video dónde se presenten los objetivos. La estrategia de comunicación posee entonces gran relevancia para el diseño de la interfaz y para la e-Accesibilidad ya que determina el estilo, la organización y la jerarquización de los componentes de la interfaz que hará el proyectista.

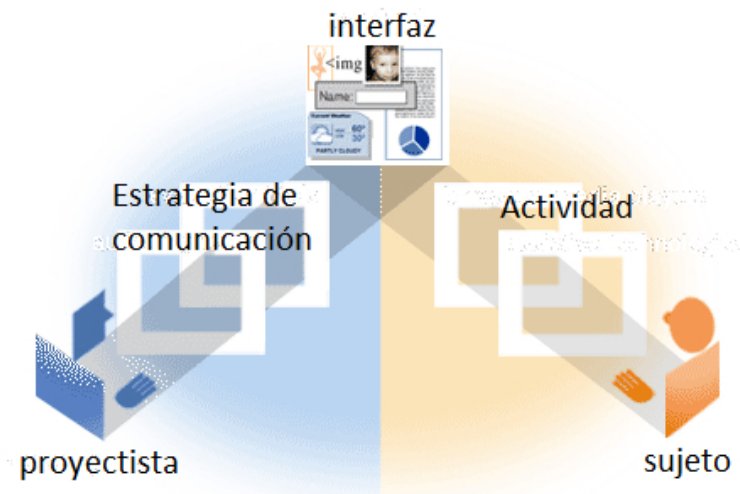

Figura 2. Modelo del enfoque comunicacional (superpuesto por la autora sobre Duffy, 2016, w3.org/WAl/intro/components.php, para resaltar la diferencia de modelos)

Por otra parte, la Teoría de la Actividad aplicada al diseño de interfaz sostiene que la interacción persona-ordenador es en realidad una actividad mediada, es decir, que el sujeto no interactúa con el ordenador sino con un objeto del mundo a través del ordenador. Por ejemplo, cuando un sujeto sigue un módulo en un MOOC, su atención no está puesta en la reproducción de los videos sino en los temas del curso que está viendo y aprendiendo. Al hablar de sujeto y no de usuario se está subrayando su capacidad de intervenir en el mundo al igual que su pertenencia a una sociedad y a una cultura que lo influencian profundamente. Esta perspectiva posee gran relevancia para el diseño de la interfaz y para la e-Accesibilidad porque el sujeto interpretará la interfaz de acuerdo a los hábitos y las convenciones que existen en el contexto de su actividad. Al hablar 
de contexto de la actividad no se refiere al conjunto de dispositivos tecnológicos que el sujeto usa para acceder a la interfaz sino a las costumbres y convenciones que existen en el entorno socio-cultural del sujeto. La Figura 2 modeliza las nociones que conforman el enfoque comunicacional propuesto.

Cabe señalar que el enfoque comunicacional hace abstracción de los componentes técnicos, ya que su foco está puesto en la comunicación humana. Esto no impide que la interfaz se materialice en componentes técnicos que se tendrán en cuenta a la hora de poner en práctica el enfoque.

\section{Ilustración de ambos enfoques}

Se mostrará sobre un caso concreto cuál es el diseño accesible propuesto por el enfoque centrado en el contenido y cuáles son los aportes que puede hacer el enfoque comunicacional. El portal Coursera (Coursera Inc., 2015) es un portal que ofrece cursos MOOC de instituciones de todo el mundo. La estrategia de comunicación en la página de inicio (Figura 3) busca persuadir al público objetivo insistiendo sobre la calidad de los cursos, su facilidad de acceso y su gratuidad. El título "Toma los mejores cursos [...]" se impone sobre los demás componentes de la interfaz gracias a su diseño visual: el fondo oscuro contrasta fuertemente con el color del texto y el tamaño de tipografía es el más grande de la página. Evidentemente este título capta la atención de una persona que puede percibir el diseño visual.
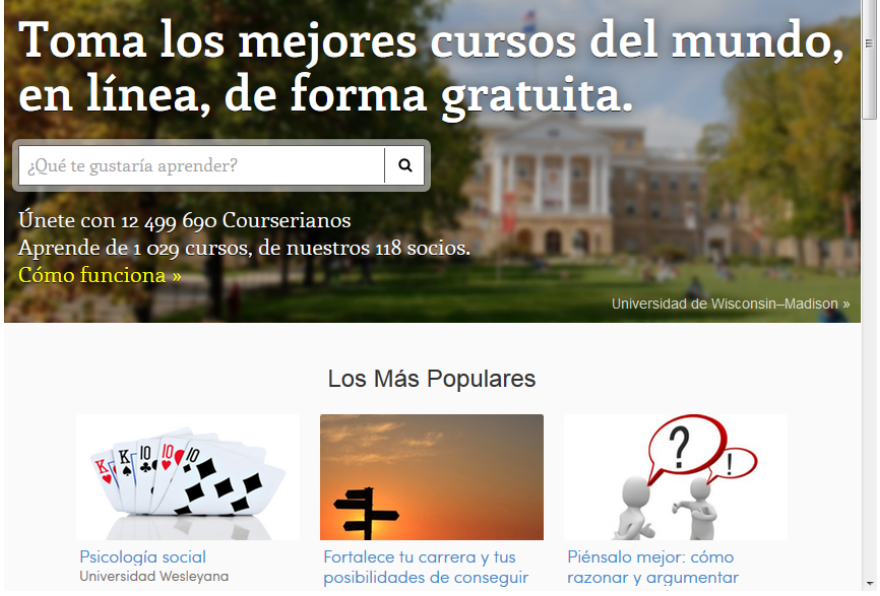

Figura 3. Página de inicio del portal Coursera (2015-04-22)

Ahora bien, si la página de inicio se lee con un lector de pantalla, el orden de lectura seguirá el orden visual de arriba hacia abajo y de izquierda a derecha. Es decir, comenzará por el logo de Coursera, seguirá con el enlace "Cursos", etc. Esto es así porque el criterio de conformidad 1.3.2 de las WCAG 2.0 sugiere que el orden de lectura del lector de pantalla respete el orden de la presentación visual. El orden de la presentación visual está dado, según el enfoque centrado en el contenido, por la direccionalidad del texto (de izquierda a derecha o de derecha a izquierda) y por las estructuras del lenguaje de marcado como las tablas o las listas. Si bien este criterio de ordenamiento no es inválido, el enfoque comunicacional aporta un criterio de más alto nivel fundado sobre la estrategia comunicacional del proyectista. El enfoque comunicacional considera que el orden de lectura del lector de pantalla debe seguir la jerarquización establecida por la estrategia de comunicación, que es en definitiva la reflejada por el diseño visual. En el ejemplo, el lector de pantalla debería comenzar por la zona de fondo oscuro, seguir por los cursos más populares y luego pasar a los enlaces del encabezado.

Concentrémonos ahora en la lista de cursos más populares. Cada curso está representado por una imagen, su nombre y el nombre de la universidad que lo auspicia. Desde el enfoque centrado en el contenido, estos tres elementos constituyen un enlace hacia el curso. La imagen de cada curso es, por lo tanto, una imagen funcional, es decir, una imagen que se usa para iniciar una acción (Leiserson, 2014, párr. 1). Según el criterio de conformidad 1.1.1 de las WCAG 2.0, cada imagen debe ir acompañada de una alternativa textual destinada a las personas que no pueden ver la imagen. En el caso de las imágenes funcionales, la WAI recomienda que la alternativa textual exprese la acción que se iniciará en lugar de describir la imagen (ibidem). Desde esta perspectiva, una alternativa textual posible para la imagen de cada curso podría ser "Ir al curso".

El enfoque comunicacional, en cambio, sugiere elaborar una alternativa accesible que conserve la estrategia de comunicación de cada imagen. La imagen del curso "Psicología social", por ejemplo, no fue elegida al azar por el proyectista. Se trata de una "trampa a la percepción" ya que el naipe diez de diamantes que debería ser rojo es en realidad negro. Esta trampa es usada por el profesor del curso como disparador para tratar el tema de la percepción selectiva. Así, una alternativa accesible para esta imagen debe cumplir el mismo rol disparador. Una solución posible sería componer una "trampa a la percepción" en la modalidad sonora. Del mismo modo, la imagen del curso "Fortalece tu carrera [...]" fue elegida por la relación metafórica que existe entre el amanecer y el inicio de una carrera profesional al 
igual que entre los paneles de ruta y las distintas opciones de empleo. Una alternativa accesible para esta imagen podría ser un sonido que esté en relación metafórica con el nombre del curso. La utilización de sonidos en la interfaz de usuario y sus modos de significar a través de la escucha causal, semántica, etc. han sido objeto de estudio en el campo de la Interacción Persona-Ordenador (Ferati, Pfaff, Mannheimer y Bolchini, 2012). Dichas investigaciones son oportunas a la hora de crear alternativas sonoras para el diseño accesible.

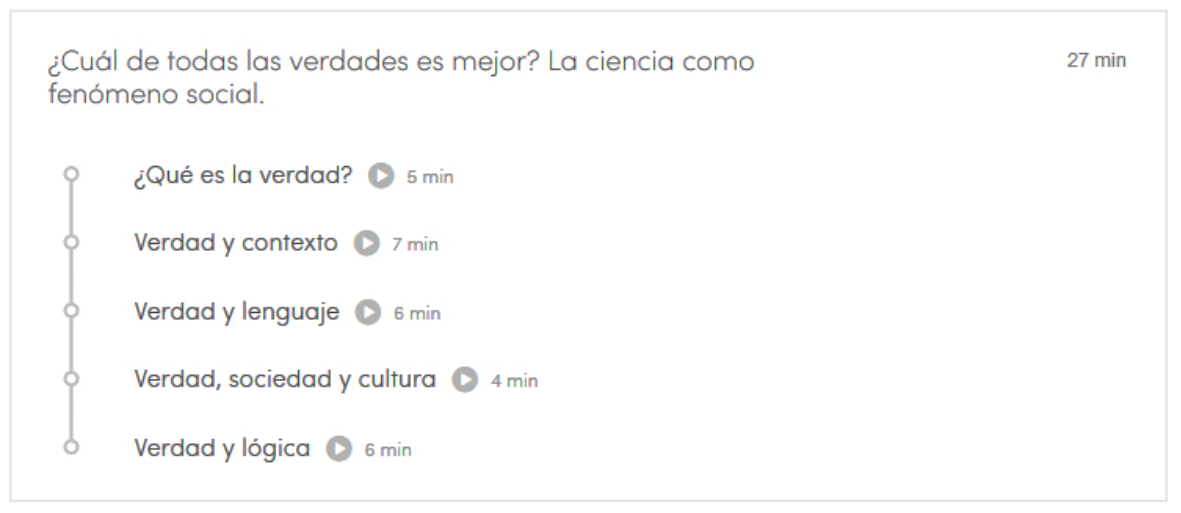

Figura 4. Consulta de un módulo del portal Coursera (2015-04-22)

Consideremos ahora una de las acciones que puede realizar el sujeto durante su actividad de aprendizaje. En la página de cada curso MOOC, existe la posibilidad de consultar la estructura y la duración de cada módulo (Figura 4). El nombre del módulo aparece en la parte superior junto a la duración total del mismo. Debajo, se listan los distintos temas del módulo, conectados por una línea vertical, con su formato y duración respectiva. Si se observa el conjunto desde el enfoque centrado en el contenido, lo que se advierte es texto y elementos gráficos. La línea vertical es un elemento gráfico que adquiere un significado por su forma, su orientación y su ubicación visual, es decir, por sus características sensoriales. Para respetar el criterio de conformidad 1.3.3 de las WCAG 2.0, se debe proveer una identificación textual de dicha línea. Ahora bien, la definición de esta identificación textual es muy relativa desde el enfoque centrado en el contenido. Es decir, no existen criterios para preferir una identificación por sobre otra entre, por ejemplo, las frases siguientes: "El módulo está compuesto por los siguientes ítems", "La duración total corresponde a la suma de las siguientes duraciones", "Los siguientes temas deben ser aprendidos según su orden de aparición". Si se adopta en cambio el enfoque comunicacional y se considera la actividad del sujeto, se tendrá en cuenta que el sujeto sigue un curso MOOC en el cual trabaja de manera autónoma y a su ritmo. Se puede argumentar entonces que la identificación que señala el orden en el cual deben aprehenderse los temas es la identificación más relevante para la actividad del sujeto. En otras palabras, el enfoque comunicacional permite elaborar una "traducción" de los elementos gráficos que resulta útil para la actividad del sujeto.

Los enlaces o hipervínculos de la interfaz son elementos que también están vinculados a la actividad del sujeto ya que posibilitan la navegación entre las distintas páginas de un sitio web. Para que un diseño sea accesible se debe prestar especial atención a la denominación de estos enlaces. El criterio de conformidad 2.4 .4 de las WCAG 2.0 sugiere que el texto de un enlace describa el propósito del enlace, esto es "La naturaleza del resultado obtenido al activar un enlace." (W3C, 2009, sec. Apéndice A: Glosario). Consideremos por ejemplo el enlace "Iniciar Sesión" que se encuentra en el encabezado de la página de inicio del portal Coursera (Figura 3). Al activar este enlace se abre una pantalla de inicio de sesión en la que se ofrecen tres posibilidades: identificarse con Facebook ${ }^{\circledR}$, identificarse con una dirección de email o recuperar una contraseña olvidada. Es decir, la denominación "Iniciar Sesión" describe adecuadamente el propósito del enlace desde el enfoque centrado en el contenido. Sin embargo, si se evalúa esta denominación desde el enfoque comunicacional, se constata que es una denominación asociada al aspecto técnico y no a la actividad del sujeto. En programación, se dice que una sesión está abierta o iniciada cuando se ingresaron un usuario y una contraseña válidos. Desde el punto de vista de la actividad, un sujeto que se encuentra en la página de inicio del portal busca iniciar sesión para comenzar o continuar un curso. Así, una denominación como "Entrar a clases" sería más representativa para el sujeto en el contexto de su actividad. 
Una vez que el sujeto se ha identificado en el portal, aparecen en el menú dos enlaces con denominaciones similares: "Cursos" y "Panel principal de cursos" (este último en el menú que se despliega a partir del nombre de usuario). El primero conduce a la lista de todos los cursos disponibles en Coursera y el segundo conduce a los cursos en los que el sujeto se ha inscrito. Desde el enfoque centrado en el contenido, la denominación de estos enlaces describe la naturaleza del resultado ya que son cursos y un panel lo que se obtiene respectivamente de cada enlace. Sin embargo, desde el enfoque comunicacional, no queda claro que desde el primer enlace el sujeto puede visualizar todos los cursos ofrecidos y eventualmente inscribirse a uno de ellos, mientras que desde el segundo enlace el sujeto puede visualizar los cursos en los que se inscribió y eventualmente continuar uno de ellos. Desde esta perspectiva, denominaciones como "Todos los cursos" y "Mis cursos" o "Inscribirme a un curso" y "Continuar un curso" serían más adecuadas.

\section{Reflexiones finales}

En las secciones que preceden se definieron e ilustraron dos maneras diferentes de concebir la e-Accesibilidad: el enfoque centrado en el contenido, que es el enfoque abogado por la WAI, y el enfoque comunicacional, que es el enfoque propuesto por el presente trabajo. No se preconiza la prevalencia de uno por sobre el otro sino más bien su complementariedad con el objetivo de alcanzar una e-Accesibilidad comunicacional más íntegra.

Mientras que el primero trata la e-Accesibilidad como una cuestión de legibilidad al contenido, el segundo trata la e-Accesibilidad como una cuestión de comunicación. Es decir, si bien los criterios de legibilidad, como el contraste de luminosidad suficiente entre un texto y su fondo (W3C, 2009, sec. 1.4.3), son imprescindibles a la hora de evaluar la e-Accesibilidad, existen otros aspectos de la comunicación, como la jerarquía que puede tener ese texto de acuerdo a la estrategia del proyectista, que deben considerarse en vistas de una verdadera e-Accesibilidad comunicacional.

La originalidad del enfoque comunicacional radica en la integración de la dimensión discursiva al modelo de la accesibilidad. Si bien los estudios anteriores propusieron ampliar el enfoque del lado del usuario considerando la usabilidad (Di Blas, Paolini y Speroni, 2004; Power, Freire, Petrie y Swallow, 2012; Rømen y Svanæs, 2011) o la experiencia de usuario (Cooper, Sloan, Kelly y Lewthwaite, 2012; Kelly y otros, 2009; Sloan y
Kelly, 2011), no se conoce una propuesta que contemple la e-Accesibilidad desde el discurso del proyectista. El enfoque comunicacional propone descubrir la estrategia de comunicación del proyectista, implícita normalmente en el diseño visual, y elaborar alternativas accesibles que conserven esta estrategia. La experiencia del usuario también se toma en cuenta cuando se contempla la actividad del sujeto.

Otra aportación del enfoque comunicacional es que permite concentrarse sobre aspectos de la comunicación que son independientes de la modalidad perceptiva de la interfaz. Tanto la estrategia comunicacional del proyectista como la actividad del sujeto son conceptos que dependen de los actores de la comunicación y no de la materialidad de los contenidos. Mientras que el enfoque centrado en el contenido trabaja a partir de la modalidad perceptiva de los contenidos, por ejemplo transforma los elementos visuales en elementos textuales para que puedan sonorizarse, el enfoque comunicacional conserva la estrategia de comunicación y la actividad del sujeto más allá de las modalidades perceptivas.

El enfoque comunicacional reconoce, al igual que la accesibilidad 2.0 (Kelly y otros, 2009), que existen diversas soluciones para el problema de la e-Accesibilidad. Tal como se mostró en los ejemplos, el enfoque comunicacional no es prescriptivo respecto de la solución que debe adoptarse, ni en cuanto a su forma, ni en cuanto a su modalidad perceptiva. El enfoque comunicacional brinda un marco teórico a partir del cual pueden inferirse alternativas de diseño accesible. Del mismo modo, el enfoque comunicacional coincide con la accesibilidad 2.0 en el punto de brindar soluciones que amplíen la participación en vez de brindar soluciones universalmente accesibles. En la accesibilidad universal subyace la idea de una solución común para todos, el enfoque comunicacional insiste en cambio sobre la individualidad de cada situación de comunicación al igual que sobre la singularidad de cada sujeto que participa.

Una limitación relativa del enfoque comunicacional con respecto al enfoque centrado en el contenido es que brinda soluciones que no pueden chequearse de manera automática. La conformidad de una página web con los criterios de las WCAG 2.0 que involucran parámetros técnicos, tal como el contraste de luminosidad, puede chequearse mediante herramientas automáticas de evaluación. En cambio, las soluciones del enfoque comunicacional sólo pueden ser evaluadas por seres humanos. El proceso de interpretación constituye la base del enfoque comunicacional y este proceso es intrínsecamente humano. 
En síntesis, el enfoque comunicacional aporta a la justificación de una nueva mirada sobre la eAccesibilidad. En este sentido se suma a los estudios que revisitan críticamente el enfoque de la WAI y hace una propuesta original fundada en estudios significativos del campo de la Interacción Persona-Ordenador. En estudios futuros se espera refinar los aspectos metodológicos del enfoque, tanto para la evaluación de la e-Accesibilidad como para el diseño accesible.

\section{Notas}

(1) Nuestra traducción. Cita original: "[...] the information that forms Web sites and Web applications: the code and markup that define the structure, presentation, and interaction, as well as text, images, and sounds that convey information to the end-user."

(2) Suelen llamarse tecnologías de apoyo o tecnologías asistivas a los programas o periféricos específicos que utilizan las personas con discapacidad para mejorar su interacción con la Web.

(3) El lector de pantalla es la tecnología asistiva que utilizan las personas con ceguera. Grosso modo, se trata de un programa capaz de convertir el texto de una interfaz en síntesis sonora.

\section{Agradecimientos}

Este trabajo se financió con un contrato doctoral de la Universidad de París 8. La autora agradece al Dr. Philippe Bootz por sus fructíferas observaciones sobre los aspectos teóricos del enfoque comunicacional, a la Dra. Paula Vera por su lectura crítica del manuscrito y al personal del Servicio de Lectura Accesible de la Biblioteca Argentina de Rosario por compartir sus experiencias en el uso de la Web.

\section{Referencias}

Bootz, P. (2007). Éléments d'analyse de l'interface sémiotique des sites Web. // I. Saleh, K. Ghdira, B. Badreddine, N. Bouhai y B. Rieder (eds.). Collaborer, Echanger, Inventer: Expériences de réseaux. Paris: Hermès Lavoisier, 2007. 107-121.

Chisholm, W. A.; Henry, S. L. (2005). Interdependent components of web accessibility. // Proceedings of the 2005 International Cross-Disciplinary Workshop on Web Accessibility. New York: ACM, 2005. 31-37. DOI: 10.1145/1061811.1061818

Cooper, M.; Sloan, D.; Kelly, B.; Lewthwaite, S. (2012). A Challenge to Web Accessibility Metrics and Guidelines: Putting People and Processes First. // Proceedings of the International Cross-Disciplinary Conference on Web Accessibility. New York: ACM, 2012. 20:1-20:4. DOI: 10.1145/2207016.2207028

Coursera Inc. (2015). Portal Coursera. https://es.coursera.org (2016-01-10).

De Souza, C. S. (2005). The semiotic engineering of humancomputer interaction. Cambridge, Mass.: MIT Press, 2005.

Di Blas, N.; Paolini, P.; Speroni, M. (2004). "Usable accessibility" to the Web for blind users. // Proceedings of 8th ERCIM Workshop "User Interfaces for All". http://goo.gl/F2TYV0 (2016-01-10).

Duffy, Michael (2016). Image. // Essential Components of Web Accessibility. S.L. Henry, ed. Copyright W3C ${ }^{\circledR}($ MIT,
ERCIM, Keio, Beihang). www.w3.org/WAl/intro/components.php

Ferati, M.; Pfaff, M. S.; Mannheimer, S.; Bolchini, D. (2012). Audemes at work: Investigating features of non-speech sounds to maximize content recognition. // International Journal of Human-Computer Studies. 70:12 (2012) 936 966. DOI: 10.1016/j.ijhcs.2012.09.003

García Gómez, J. C. (2010). Barreras para el acceso al contenido de las webs de universidades españolas. // Scire: Representación y Organización del Conocimiento. 16:1 (2010) 47-63. http://goo.gl/1eA8Sj (2016-01-10).

Henry, S. L.; Duffy, M. (2005). Essential Components of Web Accessibility. http://goo.gl/gofVgR (2016-01-10).

Kaptelinin, V. (2013). Activity Theory. // The Encyclopedia of Human-Computer Interaction (2nd ed.). http://goo.gl/PUizPk (2016-01-10).

Kelly, B.; Sloan, D.; Brown, S.; Seale, J.; Lauke, P.; Ball, S.; Smith, S. (2009). Accessibility 2.0: Next Steps for Web Accessibility // Journal of Access Services. 6:1-2 (2009) 265-294. DOI: 10.1080/15367960802301028

Laitano, M. I. (2015a). Accesibilidad web en el espacio universitario público argentino // Revista española de Documentación Científica. 38: 1 (2015) e079. DOI: 10.3989/redc.2015.1.1136

Laitano, M. I. (2015b). Semiotics Contributions to Accessible Interface Design // Proceedings of the 12th Web for All Conference. New York: ACM, 2015. 36:1-36:2. DOI: $10.1145 / 2745555.2746673$

Leiserson, A. B. (2014). Functional Images. http://goo.gl/bR4QSy (2016-01-10).

Pastor Sánchez, J. A. (2010). Bases para un Diseño Web Integral a través de la convergencia de la Accesibilidad, Usabilidad y Arquitectura de la Información. // Scire: Representación y Organización del Conocimiento. 16:1 (2010) 47-63. http://goo.gl/KDhdbS (2016-01-10).

Pignier, N.; Drouillat, B. (2004). Penser le webdesign: modèles sémiotiques pour les projets multimédias. Paris: Editions L'Harmattan, 2004.

Power, C.; Freire, A.; Petrie, H.; Swallow, D. (2012). Guidelines Are Only Half of the Story: Accessibility Problems Encountered by Blind Users on the Web. // Proceedings of the SIGCHI Conference on Human Factors in Computing Systems. New York: ACM, 2012. 433-442. DOI: 10.1145/2207676.2207736

Rabardel, P. (1995). Les hommes et les technologies: approche cognitive des instruments contemporains. Paris: Armand Colin, 1995.

Rømen, D.; Svanæs, D. (2011). Validating WCAG versions 1.0 and 2.0 through usability testing with disabled users. // Universal Access in the Information Society. 11:4 (2011) 375-385. DOI: 10.1007/s10209-011-0259-3

Scolari, C. (2009). The sense of the interface: Applying semiotics to HCl research. // Semiotica. 177 (2009) 1-27. DOI: 10.1515/semi.2009.067

Sloan, D.; Kelly, B. (2011). Web accessibility metrics for a post digital world. // Website Accessibility Metrics symposium. http://goo.gl/ekFGRr (2016-01-10).

W3C. (2009). Web Content Accessibility Guidelines (WCAG) 2.0. http://goo.gl/VPLpkl (2016-01-10).

WebAIM. (2009). Survey of Preferences of Screen Readers Users. http://goo.gl/ZxKr2L (2016-01-10).

Enviado: 2015-01-10. Segunda versión: 2016-04-20. Aceptado: 2016-05-11. 
